\title{
Simultaneous Ballistic Deficit Immunity and Resilience to Parallel Noise Sources: A New Pulse Shaping Technique ${ }^{1}$
}

\author{
L. Fabris ${ }^{2}$, J. A. Becker ${ }^{3}$, F. S. Goulding ${ }^{2}$, N. W. Madden ${ }^{2}$ \\ ${ }^{2}$ E. O. Lawrence Berkeley National Laboratory, 1 Cyclotron Road, Berkeley, California 94720 \\ ${ }^{3}$ Lawrence Livermore National Laboratory, 7000 East Ave., Livermore, California 94550
}

\begin{abstract}
A new and different time variant pulse processing system has been developed based on a simple CR-RC filter and two analog switches. The new pulse processing technique combines both ballistic deficit immunity and resilience to parallel noise without a significant compromise to the low energy resolution, generally considered a mutually exclusive requirement. The filter is realized by combining two different pulse-shaping techniques. One of the techniques creates a low rate of curvature at the pulse peak, which reduces ballistic deficit, while the second technique increases the tolerance to low frequency noise by modifying the noise history. Several experimental measurements are presented, including tests on a co-planar grid CdZnTe detector. Improvements on both the resolution and line shape are shown for the $662 \mathrm{keV}$ line of ${ }^{137} \mathrm{Cs}$
\end{abstract}

\section{INTRODUCTION}

Chargecollection is still a significant problem in some solid-state detector. For example, spectrometers utilizing wide band gap compound semiconductors such as CdTe and CdZnTe (CZT) are being developed for use at or near room temperature, but their performance is still limited by charge transport imperfections [1], [2]. In fact, when the collection of one type of carrier (holes in CZT) shows a marked difference compared to the other, then the charge pulse collected at the electrodes will have different shapes based upon the location in the detector where the charge pairs where created. This is especially bothersome at higher energies (above $100 \mathrm{keV}$ ) because of the longer interaction length of the more energetic photons [3]. When the detector material presents different mobility and trapping phenomena for electrons and holes, the resulting image charge induced on the collection electrode has a shape that depends on how far from such electrode the interaction took place [4]. Moreover, high energy photons also present Compton scattering phenomena that introduce complex structure in the image charge. Several solutions for different types of detectors, made from compound semiconductors, have been developed in order to compensate for the limitations mentioned above [5]-[8]. One of the more recent electrode structures, the co-planar grid [6], minimizes these imperfections, however, fluctuations in the charge collection processes are still present. These fluctuations are seen by the signal processing electronics as a distribution in rise times at the output of the charge preamplifier. Rise time fluctuations manifest themselves as "ballistic deficit" when convolved with the transfer function of a pulse shaping network [9].

\footnotetext{
${ }^{1}$ This work was performed under the auspices of the Department of Energy under contract No. W-7405-ENG-48.
}

The extent to which the ballistic deficit influences the resolution and line shape obtainable from a spectroscopy system depends upon the tolerance of a given pulse shaping network to fluctuations in the input rise time and to the relative ratio fluctuation/peaking time. It is intuitive in fact, that the lower the rate of curvature at the peak of the step response of a pulse shaping network, the higher the immunity to ballistic deficit [10].

It can be demonstrated that several pulse shaping networks are very tolerant to ballistic deficit [10], therefore, in principle the problem can be minimized by using electronic techniques. However, in many practical applications, the solutions applicable to reduce ballistic deficit cannot be exploited in all of their potential, due to other conflicting needs. For example, often a practical solution for the mitigation of ballistic deficit is to simply choose a long(er) peaking time (where "long" is compared to the rise time at the output of the charge preamplifier and to its spread). It is also known that a long peaking time is usually associated with signals with an increased area and this implies degradation in electronic resolution due to low frequency noise contributions (both parallel and $1 /|f|$ ) [4] and the consequential worsening of rate-handling capabilities of the pulse processing system. It is clear that in order to design a pulse shaping system immune to ballistic deficit one generally has to trade rate for resolution and tolerate the accentuation of low frequency noise terms [8].

From a similar observation, we implemented our design of a time-variant pulse shaper able to offer ballistic deficit mitigation without incurring a substantial penalty in the electronic resolution and rate capability.

\section{CIRCUIT}

Among the time-invariant circuits, a CR-RC shaping network has a transfer function such that the resulting shape is very tolerant to ballistic deficit for a given peaking time [10][12], due to the fact that its rate of curvature at the peak is very low. This statement is illustrated in Fig. 1, where the response of different shaping networks, with a nominal $1 \mu \mathrm{s}$ peaking time, to an infinitely steep input step and to a $500 \mathrm{~ns}$ rise time step are shown. This represents, for example, the real case of a thick $(>2 \mathrm{~mm})$ CZT detector irradiated by a ${ }^{137} \mathrm{Cs}$ source $\left(E_{\gamma}=\right.$

$662 \mathrm{keV}$ ). In Fig. 1 we compare, on an arbitrary vertical scale, the CR-RC shaping network to other two shaping networks that are among the most commonly used in nuclear spectroscopy: the so-called $\sin ^{4}$ and $\sin ^{6}$ shaping networks. The amplitude of the responses to an ideal pulse (infinitesimal rise time) is normalized to 1 and the numbers in parenthesis show the time vs. amplitude value of the waveforms at the 
maximum. Note, that for a spread of $0.5 \mu \mathrm{s}$ in the input risetime (equal to $50 \%$ of the nominal peaking time), the CR$\mathrm{RC}$ shaper suffers only a $1 \%$ degradation in the value of the peak amplitude, while the other two shapers show respectively a degradation of $4.7 \%$ and $18 \%$. In other words, in order to obtain the same ballistic deficit immunity (1\%) from the sinusoidal shapers, one should choose a peaking time of $2.5 \mu \mathrm{s}$ for the $\sin ^{4}$ shaper and $3.3 \mu$ s for the $\sin ^{6}$ shaper. The increased peaking time not only has a negative impact on the rate, but also on the resolution in presence of low frequency noise.

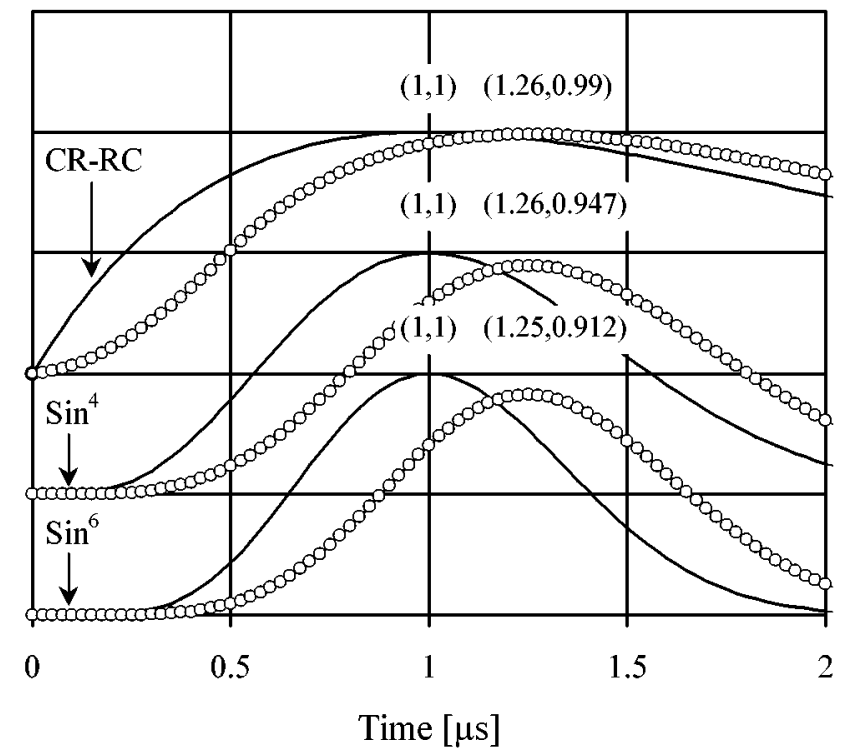

Fig. 1. Ballistic deficit immunity of a CR-RC shaping network, compared to two commonly used shapers.

The drawback in using this shaping network is that the pulse baseline-to-baseline width to peaking time ratio is very high. This precludes the use of such a filter for many applications for two reasons. The input rate at which a system based on this pulse shaping network can operate is low. In the presence of significant low frequency noise, frequently encountered in CZT detectors operated at room temperature, there is also a loss of resolution (low frequency noise contribution is proportional to the total area of the weighting function of a shaper [8]).

In order to overcome the problems just mentioned and still take advantage of the features offered by the CR-RC pulse shaping network, a time variant approach is taken.

Our idea is to present a certain CR-RC type of transfer function only when a signal (gamma-ray) is present, and a substantially faster response in absence of signal. The reason for a faster response during the absence of signal is that the contribution of low frequency noise and instabilities in the baseline are reduced by truncating the noise history. As can be readily understood using Goulding's approach to signal processing [13] there is some penalty to pay. Namely, when the circuit is in the "fast" mode, the contribution of high frequency noise is higher than in the "slow" mode. However, in presence of large low frequency noise this is only a little price to pay, as it will be shown later.

We chose to implement the shaping network through a simple CR-RC cascade in which the time constant associated to each block can be switched to a long or to a shorter value depending upon the presence of a signal at the input. A simplified schematic block is shown in Fig. 2.

The operational amplifiers, together with the resistors $R, R_{1}$, $\mathrm{R}_{2}$ and the capacitors $\mathrm{C}$, implement the differentiation and the integration stages. The high-quality analog delay allows the circuit block labeled "Switch Control Logic" to recognize a valid signal at the input and to correctly actuate the switches over all the rise time values of interest. The implementation of the control logic block could be as simple as a fast discriminator plus delays (such as those obtainable from a oneshot) or as elaborated as the peak detector described in [14]. The value of the analog delay must be at least equal to the longest rise time of the input signal. This way, the discriminators in the control logic can recognize the presence of a gamma-ray signal and sequence the switches. It is worth pointing out that in our circuit the switches are in the signal path only when there is no valid input. In fact, when a valid input is recognized by the control logic, the switches are toggled from the "ON" (or closed) position to the "OFF" (or open) position. The switches remain in the OFF position until a peak detector finds the most positive excursion (peak). Once the peak is found, the peak detector returns the switches to the ON position. Using the circuit described in [14], we are sure that the peak is always identified without uncertainties, even when large ballistic deficit effects are present (see Fig. 1) because this peak detector exactly identifies the sign reversal of a current into a capacitor. For a unipolar shaped signal, this occurs at the peak exactly. The timing of the filter is selfadaptive to the true peak position. It is important to note that our choice of switch implementation removes all of the uncertainties and instabilities usually associated with analog switches. The switches are absent during the analog measurement.

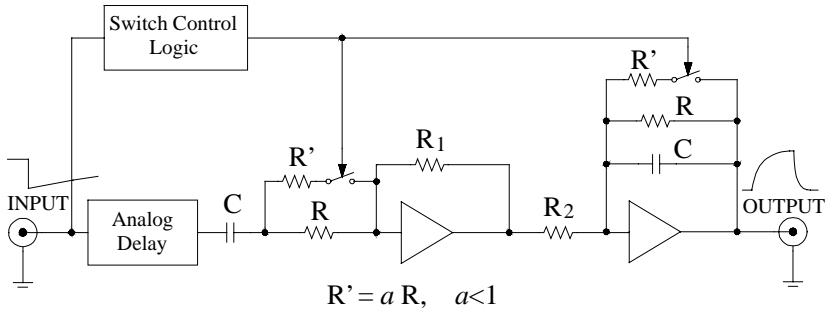

Fig. 2: Simplified block diagram of the switched shaper.

The multiplier $a$ (see Fig. 2) is such that, the time constants with switches on $\left(\tau_{O N}\right)$ or off $\left(\tau_{O F F}\right)$ are related as follow:

$$
\tau_{\text {ON }}=\frac{a}{1+a} \cdot \tau_{\text {OFF }}=\eta \cdot \tau_{\text {OFF }}, \quad(\eta \leq 1)
$$


In (1) we introduced $\eta$ for simplicity only. The choice of the fraction $\eta$ is very important for the global behavior of the shaper. A $\eta=1$ is the same as the unswitched CR-RC, while for $\eta<<1$ the shorter time constant approaches zero. The best way of choosing the appropriate $\eta$ is to study the noise response of the corresponding noise residual function [8] associated with the particular choice. By using Goulding's numerical methods we were able to exctract the noise index for series - $\left\langle N_{\Delta}^{2}\right\rangle$ and parallel $-\left\langle N_{S}^{2}\right\rangle$ noise normalized to 1 $\mu$ s peaking time, plus the factor of merit (FOM) of the shaper [8]. Table I shows the results obtained, compared with some commonly used time-invariant shapers.

TABLE I

NOISE INDEXES AND FACTORS OF MERIT OF THE SWITCHED SHAPER IN COMPARISON WITH COMMONLY USED FILTERS

\begin{tabular}{clccc}
\hline \multicolumn{1}{c}{$\begin{array}{c}\text { Type } \\
\text { Of } \\
\text { Shaper }\end{array}$} & $\left\langle N_{S}^{2}\right\rangle$ & $\left\langle N_{\Delta}^{2}\right\rangle$ & FOM \\
\hline & $\eta=1$ & 1.847 & 1.847 & 1.847 \\
Switched & $\eta=0.5$ & 1.22 & 2.10 & 1.60 \\
CR-RC & $\eta=0.2$ & 0.85 & 2.85 & 1.55 \\
& $\eta=0.1$ & 0.72 & 4.10 & 1.72 \\
& $\eta=0.05$ & 0.66 & 6.60 & 2.10 \\
Sin $^{4}$ & - & 0.70 & 2.49 & 1.31 \\
Sin $^{6}$ & - & 0.64 & 2.61 & 1.29 \\
Pseudo- & $500 \mathrm{~ns}$ & 1.17 & 2.00 & 1.53 \\
Trapezoid & flat-top & & & \\
\hline
\end{tabular}

From Table I we see that as stated before, the behavior of the time-variant shaper low frequency noise improves significantly as the fraction $\eta$ decreases. At the same time, the high frequency noise index degenerates to undesirable levels. Some time-invariant shapers have still better performance both for high and low frequency noise, but the numbers in Table I do not take into account ballistic deficit sensitivity. After different tests we decided to adopt $\eta=0.1$ for our experimental measurements, since this value of the parameter led to the best results in presence of large low frequency noise terms. With this choice, the ballistic deficit remains unchanged from the CR-RC filter, while the return to the baseline is dramatically improved (Fig. 3), opening new possibilities in high counting rate applications.

\section{MEASUREMENTS}

Several experimental measurements have been performed in order to prove our hypothesis previously stated about low frequency noise performance and ballistic deficit tolerance.

Table I shows a ratio of 2.39 between the step noise index of the CR-RC shaper and that of the switched shaper. This means that when the parallel noise is the dominant noise contribution, a similar ratio must be observed in the total noise power at the output of the shapers, or a factor of 1.6 in the output voltage noise. To verify this statement we implemented a measurement in which the shapers where fed by the same low noise charge preamplifier (input noise spectral density $<1 \mathrm{nV} / \sqrt{\mathrm{Hz}}$ in the frequency range of interest and negligible current noise) with an AC coupled resistor between its input and ground. The value of the resistor (100 Mohm) guarantees a constant spectral noise density of $1.3 \mu \mathrm{V} / \sqrt{\mathrm{Hz}}$ at the preamplifier's input; this is more than enough to ensure that over the range of frequency of interest, this noise contribution dominates. The output response of both the conventional CR$\mathrm{RC}$ shaper and of the switched CR-RC shaper, stimulated with a noiseless electronic test pulser, has been measured at $5 \mu \mathrm{s}$ peaking time with the aid of a multi-channel analyzer.

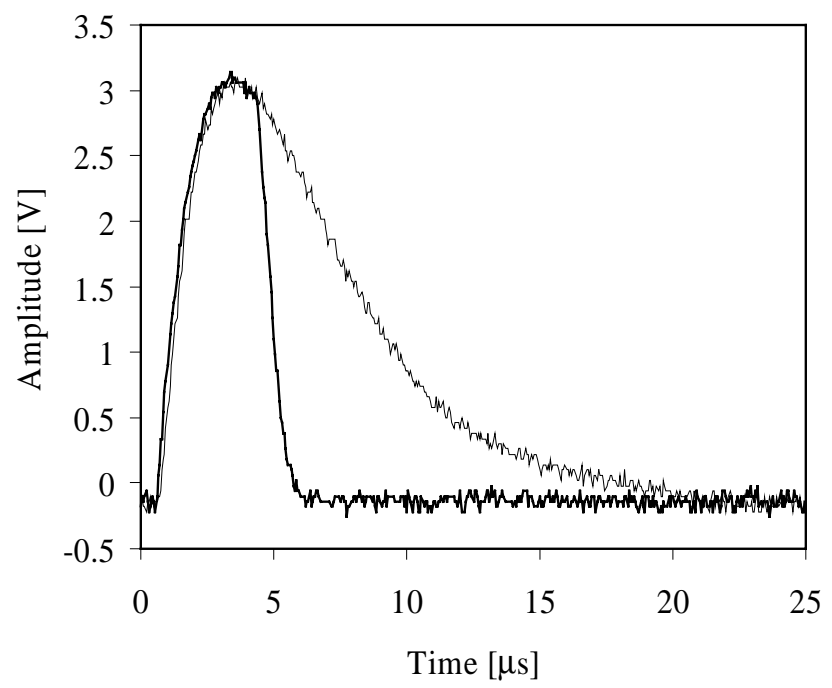

Fig. 3: Step response of the switched shaper ( $\eta=0.1$ ), compared to an ordinary CR-RC shaper.

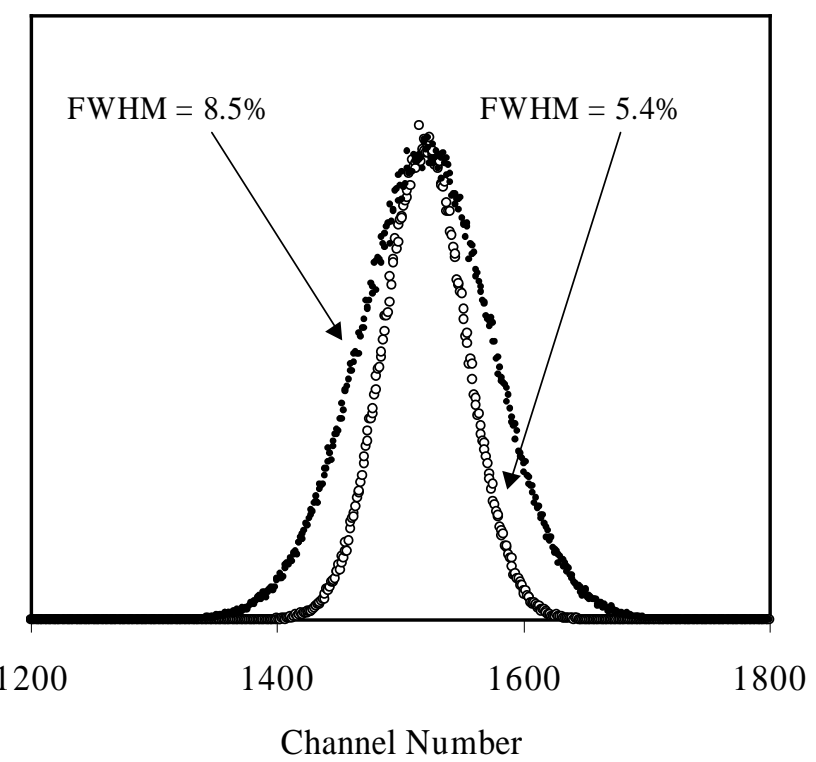

Fig. 4: Total output noise measured at the output of a CR-RC shaper (solid dots) and at the output of the switched shaper (white dots) at a peaking time of $5 \mu \mathrm{s}$. 


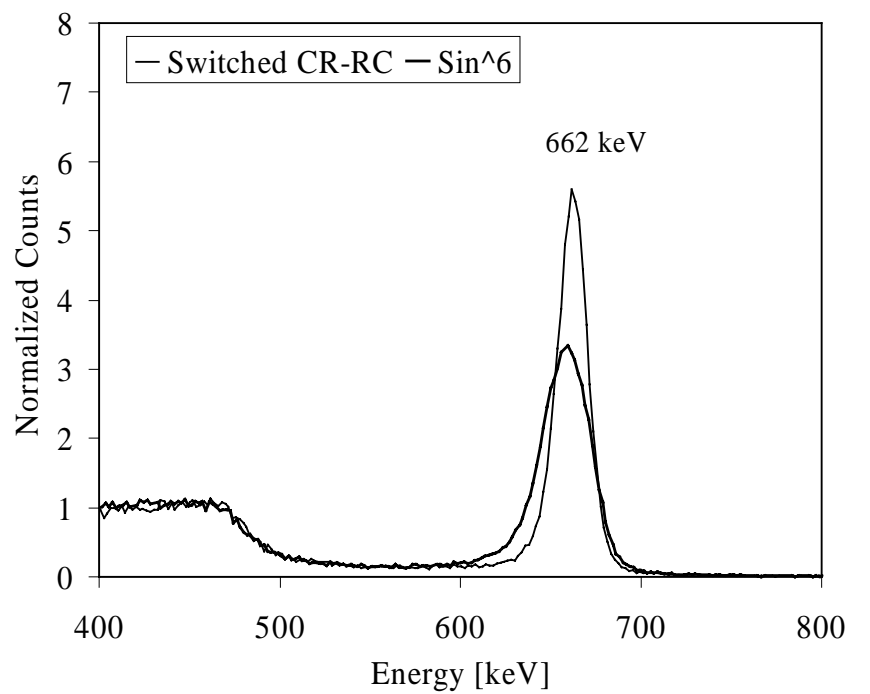

Fig. 5: Spectrum of a ${ }^{137} \mathrm{Cs}$ source taken using the switched CRRC shaper in comparison with a commercial pseudo-Gaussian shaper. Both measurements were taken at $1.5 \mu \mathrm{s}$ peaking time and $-1300 \mathrm{~V}$ detector bias.

The amplitude distribution is shown in Figure 4 in arbitrary channel units. The measured ratio of the two full widths at half maximum (FWHM) is 1.6, in very good agreement with theory.

Another measurement shows the ballistic deficit tolerance of the switched CR-RC shaper versus a $\sin ^{6}$ shaper (Table II). We used in this case two elements of a four-element CZT detector with co-planar grid measuring both the best- and the worstperforming channel. A comparison has been made between the switched CR-RC shaper and a commercial state-of-the-art, six poles, pseudo-Gaussian shaper, both set at $1.5 \mu \mathrm{s}$ peaking time. This peaking time is such that, although mitigated by the co-planar grid, ballistic deficit phenomena are still evident. In this case we also swept the detector bias $200 \mathrm{~V}$ around the nominal $-1300 \mathrm{~V}$ to investigate the efficiency of the ballistic deficit correction. The results for the worst and best performing channels are shown respectively in Table II(a) and Table II(b).

From the data of Table II, it can be seen that there is a dramatic improvement in the system resolution whenever ballistic deficit effects are present. As an example, Fig. 5 shows a ${ }^{137} \mathrm{Cs}$ spectrum taken from the poorer channel at a bias of $-1300 \mathrm{~V}$ and $1.5 \mu \mathrm{s}$ peaking time. The FWHM for the 662 $\mathrm{keV}$ line was respectively $32.9 \mathrm{keV}$ and $18.3 \mathrm{keV}$.

The two spectra are normalized to the area of the $662 \mathrm{keV}$ line of ${ }^{137} \mathrm{Cs}$. This means that the same number of events was acquired in both measurements

The improvement introduced by the higher tolerance to ballistic deficit is reflected directly into the peak shape: in the case of our time variant filter the energy peak has a more defined gaussian shape. In fact, while the full width at a tenth of maximum (FWTM) is $67 \mathrm{keV}$ for the case of the commercial shaper, in our case the FWTM is only $40 \mathrm{keV}$. The improvement in the resolution is such that the Compton to peak ratio is more favorable in the case of the switched filter (in this case $\sim 60 \%$ improvement was observed) resulting in a shorter acquisition time for a given statistics.

TABLE II

RESOLUTIONS

\begin{tabular}{clll}
\hline $\begin{array}{c}\text { Det. Bias } \\
\text { [V] }\end{array}$ & \multicolumn{1}{c}{ Shaper } & $\begin{array}{c}\text { Resol. }{ }^{241} \mathrm{Am} \\
{[\mathrm{keV}]}\end{array}$ & $\begin{array}{c}\text { Resol. }{ }^{137} \mathrm{Cs} \\
{[\mathrm{keV}]}\end{array}$ \\
\hline \multirow{2}{*}{-1100} & Sin $^{6}$ & 8.9 & 47 \\
& Switched CR-RC & 10 & 21.8 \\
-1200 & Sin $^{6}$ & 9.3 & 37.1 \\
& Switched CR-RC $^{6}$ & 9.6 & 20.5 \\
-1300 & Sin $^{6}$ & 8.8 & 32.9 \\
& Switched CR-RC $^{6}$ & 8.2 & 18.3 \\
-1400 & Sin $^{6}$ & 10.5 & 27 \\
& Switched CR-RC $^{6}$ & 9.4 & 19.3 \\
-1500 & Sin $^{6}$ & 9.9 & 24.4 \\
& Switched CR-RC & 10.3 & 20.5 \\
\hline
\end{tabular}

(a)

\begin{tabular}{|c|c|c|c|}
\hline $\begin{array}{l}\text { Det. Bias } \\
{[\mathrm{V}]}\end{array}$ & Shaper & $\begin{array}{l}\text { Resol. }{ }^{241} \mathrm{Am} \\
{[\mathrm{keV}]}\end{array}$ & $\begin{array}{c}\text { Resol. }{ }^{1{ }^{17}} \mathrm{Cs} \\
{[\mathrm{keV}]}\end{array}$ \\
\hline \multirow[b]{2}{*}{-1100} & $\operatorname{Sin}^{6}$ & 8.1 & 42 \\
\hline & Switched CR-RC & 9.2 & 18 \\
\hline \multirow{2}{*}{-1200} & $\operatorname{Sin}^{6}$ & 7.9 & 32.8 \\
\hline & Switched CR-RC & 9.2 & 16 \\
\hline \multirow[b]{2}{*}{-1300} & $\operatorname{Sin}^{6}$ & 8.4 & 28.4 \\
\hline & Switched CR-RC & 9.7 & 18.1 \\
\hline \multirow{2}{*}{-1400} & $\operatorname{Sin}^{6}$ & 8.4 & 22 \\
\hline & Switched CR-RC & 9.4 & 15.1 \\
\hline \multirow{2}{*}{-1500} & $\operatorname{Sin}^{6}$ & 8.2 & 16.4 \\
\hline & Switched CR-RC & 9.6 & 15.9 \\
\hline
\end{tabular}

(b)

Resolution of ${ }^{241} \mathrm{Am}\left(E_{\gamma} \approx 60 \mathrm{keV}\right)$ and ${ }^{137} \mathrm{Cs}\left(E_{\gamma} \approx 662 \mathrm{keV}\right)$ lines as measured in the worst channel (a) and in the best channel (b) of a four-channel CZT detector. The peaking time was $1.5 \mu \mathrm{s}$ for both shapers and the label " $\operatorname{Sin}^{6 "}$ refers to the state-of-the-art commercial shaper used. The rate was $\sim 10 \mathrm{kHz}$.

It is important to notice that, when the ballistic deficit effects become less important (see bias conditions at $-1500 \mathrm{~V}$ ), the switched shaper still has better rate performance, because its total time over the baseline is shorter than that of the pseudo-Gaussian filter for the same peaking time.

\section{ACKNOWLEDGMENT}

The authors would like to acknowledge Donald Landis and PierFrancesco Manfredi of Lawrence Berkeley National Laboratory for the many insightful suggestions and comments. The authors are also indebted to Paul Luke, of Lawrence Berkeley National Laboratory, for the generous loan of the CZT detectors used to generate the experimental data in this paper. 


\section{REFERENCES}

[1] P. Fougeres, P. Siffert, M. Hageali, J. M. Koebel, R. Regal, "CdTe and $\mathrm{Cd}_{1-\mathrm{x}} \mathrm{Zn}_{\mathrm{x}} \mathrm{Te}$ for nuclear detectors: facts and fictions", Nucl. Instr. and Meth. in Phys. Res. A 428 (1999) 38-44.

[2] K. Hitomi, T. Murayama, T. Shoji, T. Suehiro, Y. Hiratate, "Improved spectrometric characteristics of thallium bromide nuclear radiation detectors", Nucl. Instr. and Meth. in Phys. Res. A 428 (1999) 372-378.

[3] F.S. Goulding, R.H. Pehl, "Semiconductor Radiation Detectors", Nuclear Spectroscopy and Reactions, Part. A, 289-343, J. Cerny Editor, Academic Press, New York, 1974.

[4] F.S. Goulding, D.A. Landis, "Signal processing for semiconductor detectors", IEEE Trans. on Nucl. Sci., vol. NS-29, no. 3, June 1982.

[5] F.S. Goulding, D.A. Landis, "Signal processing for semiconductor detectors", IEEE Trans. on Nucl. Sci., vol. NS-29, no. 3, June 1982.

[5] O. Frisch, British Atomic Energy Report BR-49 (1944).

[6] P.N. Luke, Appl. Phys. Lett. 65, 2884 (1994).

[7] S.M. Hinshaw, D.A. Landis, IEEE Trans. on Nucl. Sci., vol. NS-37, no. 1, 1990.
[8] F.S. Goulding, D. A. Landis, N. W. Madden, M. Maier, H. Yaver, "GAMMASPHERE - Elimination of Ballistic Deficit by Using A Quasi-Trapezoidal Pulse Shaper", IEEE Trans. on Nucl. Sci., vol. 41, no. 4, August 1994.

[9] G. F. Knoll, Radiation Detection and Measurement, 2nd ed., John Wiley \& Sons, New York, 1989, p. 606.

[10]E. Fairstein, "Linear Unipolar Pulse-Shaping Networks: Current Technology", IEEE Trans. on Nucl. Sci., vol. 37, no. 2, April 1990.

[11] AB. Gillespie, Signal, Noise and Resolution in Nuclear Counter Amplifiers, McGraw-Hill Book Co., New York, 1953.

[12]B.W. Loo et al., "Ballistic Deficits in Pulse Shaping Amplifiers", IEEE Trans. Nucl. Sci., Vol. NS-35, No. 1, February 1988.

[13]F.S. Goulding, D.A. Landis, "Semiconductor Detector Spectrometer Electronics", Nuclear Spectroscopy and Reactions, Part. A, 413-481, J. Cerny Editor, Academic Press, New York, 1974.

[14] L. Fabris, P. G. Allen, J. J. Bucher, N. M. Edelstein, D. A. Landis, N. W. Madden, D. K. Shuh, "Fast Peak Detector Stretchers for Use in XAFS Applications", IEEE Trans. on Nucl. Sci., vol. 46, no. 6, December 1999. 